\title{
TESTING THE COSMIC MICROWAVE BACKGROUND DATA FOR SYSTEMATIC EFFECTS
}

\author{
Louise M. Griffiths and Charles H. Lineweaver \\ Department of Astrophysics and Optics, School of Physics, University of New South Wales, Sydney NSW 2052, Australia \\ Received 2003 July 28; accepted 2003 November 18
}

\begin{abstract}
Under the assumption that the concordance $\Lambda$ cold dark matter (CDM) model is the correct model, we test the cosmic microwave background (CMB) anisotropy data for systematic effects by examining the bandpass temperature residuals with respect to this model. Residuals are analyzed as a function of angular scale $l$, Galactic latitude, frequency, calibration source, instrument type, and several other variables that may be associated with potential systematic effects. Our main result is that we find no significant systematic errors associated with these variables. However, we do find marginal evidence for a trend associated with Galactic latitude indicative of Galactic contamination.
\end{abstract}

Subject headings: cosmic microwave background - cosmology: observations - methods: data analysis

On-line material: color figures, machine-readable table

\section{INTRODUCTION}

The cosmic microwave background (CMB) power spectrum is a particularly potent probe of cosmology. As long as the systematic errors associated with these observations are small, the detected signal has direct cosmological importance. The ever-tightening network of constraints from $\mathrm{CMB}$ and nonCMB observations favors a concordant $\Lambda$ cold dark matter (CDM) model that is commonly accepted as the standard cosmological model (Table 1). Since the anisotropy power spectrum is playing an increasingly large role in establishing and refining this model, it is crucial to check the CMB data for possible systematic errors in as many ways as possible.

Systematic errors and selection effects are notoriously difficult to identify and quantify. Individual experimental groups have developed various ways to check their CMB observations for systematic effects (e.g., Kogut et al. 1996; Miller et al. 2002), including the use of multiple calibration sources, multiple frequency channels, and extensive beam calibrating observations. Internal consistency is the primary concern of these checks.

Testing for consistency with other CMB observations is another important way to identify possible systematic errors. When the areas of the sky observed overlap, this can be done by comparing CMB temperature maps (e.g., Ganga et al. 1994; Lineweaver et al. 1995; Xu et al. 2001). When similar angular scales are being observed, one can compare power spectra (e.g., Sievers et al. 2003, Fig. 11). A prerequisite for the extraction of useful estimates for cosmological parameters from the combined CMB data set is the mutual consistency of the observational data points (Wang, Tegmark, \& Zaldarriaga 2002); the best fit must also be a good fit. Wang et al. (2002) and Sievers et al. (2003) have recently explored the consistency of various $\mathrm{CMB}$ observations with respect to power spectrum models and concluded that the CMB fluctuation data are consistent with several minor exceptions.

Although individual observational groups vigorously test their data sets for systematic errors, the entire CMB observational data set has not yet been collectively tested. Here we check for consistency of the concordance model (Table 1) with respect to possible sources of systematic error. Under the assumption that the concordance model is the correct model (i.e., more correct than the best fit to the CMB data alone), we explore residuals of the observational data with respect to this model to see if any patterns or outliers emerge. We attempt to identify systematic errors in the data that may have been ignored or only partially corrected for.

With only a few independent band power measurements the usefulness of such a strategy is compromised by low number statistics. However, we now have hundreds of band power measurements on scales of $2<l<2000$ from over two dozen autonomous and semiautonomous groups. There are enough CMB fluctuation detections from independent observations that subtle systematic effects could appear above the noise in regression plots of the data residuals. This is particularly the case when one has a better idea of the underlying model than provided by the CMB data alone.

The history of the estimates of the position of the CMB dipole illustrates the idea. Once a relatively precise direction of the dipole was established, the positional scatter elongated in the direction of the Galactic center could be distinguished unambiguously from statistical scatter and more reliable corrections for Galactic contamination could be made (Lineweaver 1997, Fig. 2). We aim to ascertain whether the use of the concordance model as a prior can help to separate statistical and systematic errors in the CMB anisotropy data. In $\S 2$ we discuss constraints on cosmological parameters, the current concordance model, and how simultaneously analyzing combinations of independent observational data sets can tighten cosmological constraints. Our analytical methodology is detailed in $\S 3$. In $\S 4$ possible sources of systematic uncertainty are discussed. In $\S \S 5$ and 6 our results are discussed and summarized.

\section{THE CONCORDANCE COSMOLOGY}

\subsection{Observational Concordance}

The CMB has the potential to simultaneously constrain a number of cosmological parameters that are the ingredients of the hot big bang model. Unfortunately, particular parameter combinations can produce indistinguishable $C_{l}$ spectra (Efstathiou \& Bond 1999). For example, cosmological models with different matter content but the same geometry can have nearly identical power spectra. Such model degeneracies limit parameter extraction from the $\mathrm{CMB}$ alone. 
TABLE 1

$1 \sigma$ Cosmological Parameter Constraints from Five Analyses

\begin{tabular}{|c|c|c|c|c|c|c|c|c|c|c|c|}
\hline \multirow[b]{2}{*}{ Parameter } & \multicolumn{2}{|c|}{ Efstathiou et al. (2002) } & \multicolumn{2}{|c|}{ Sievers et al. (2003) } & \multicolumn{2}{|c|}{ Lewis \& Bridle (2002) } & \multicolumn{2}{|c|}{ Wang et al. (2003) } & \multicolumn{2}{|c|}{ Spergel et al. (2003) } & \multirow[b]{2}{*}{$\begin{array}{c}\Lambda \text { CDM } \\
\text { CONCORDANCE }\end{array}$} \\
\hline & CMB Alone & $\begin{array}{c}+2 \mathrm{dFGRS} \\
+\mathrm{BBN}\end{array}$ & CMB Alone & + Priors $^{\mathrm{a}}$ & $\mathrm{CMB}+$ Priors $^{\mathrm{b}}$ & $+2 \mathrm{dFGRS}$ & $\begin{array}{l}\text { CMB + } \\
\text { Flat Prior }\end{array}$ & $+2 \mathrm{dFGRS}$ & $\begin{array}{l}\mathrm{CMB}(\mathrm{TT}+\mathrm{TE}) \\
+ \text { Flat Prior }\end{array}$ & $+2 \mathrm{dFGRS}+\mathrm{Ly} \alpha$ & \\
\hline$\Omega_{k} \ldots \ldots \ldots \ldots \ldots \ldots$ & $-0.04_{-0.32}^{+0.05}$ & $-0.013_{-0.019}^{+0.027}$ & $-0.05 \pm 0.05$ & $(0.00)$ & $(0.00)$ & $(0.00)$ & $(0.00)$ & $(0.00)$ & $(0.00)$ & $(0.00)$ & 0.0 \\
\hline$\Omega_{\Lambda} \ldots \ldots \ldots \ldots \ldots$ & $0.43^{+0.23}$ & $0.73 \pm 0.04$ & $0.54_{-0.13}^{+0.12}$ & $0.70_{-.03}^{+.02}$ & $0.72 \pm 0.06$ & $0.71 \pm 0.04$ & $0.71 \pm 0.11$ & $0.72 \pm 0.09$ & $0.76_{-0.06}^{+0.05}$ & $0.74_{-0.04}^{+0.03}$ & 0.74 \\
\hline 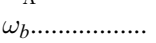 & $0.020_{-0.002}^{+0.013}$ & $0.020 \pm 0.001$ & $0.023 \pm 0.003$ & $0.024_{-0.003}^{+0.0002}$ & $0.022 \pm 0.001$ & $0.022 \pm 0.001$ & $0.023 \pm 0.003$ & $0.024 \pm 0.003$ & $0.023 \pm 0.001$ & $0.0226 \pm 0.0008$ & 0.0226 \\
\hline$\omega_{c} \ldots \ldots \ldots \ldots \ldots \ldots$ & $0.13_{-0.05}^{+0.03}$ & $0.10_{-0.01}^{+0.02}$ & $0.13_{-0.02}^{+0.03}$ & $0.12_{-0.01}^{+0.01}$ & & & $0.112 \pm 0.014$ & $0.115 \pm 0.013$ & $0.11_{-0.04}^{+0.06}$ & $0.11 \pm 0.03$ & 0.11 \\
\hline$\omega_{d} \ldots \ldots \ldots \ldots \ldots \ldots \ldots$ & $\ldots$ & $\ldots$ & $\ldots$ & $\ldots$ & $0.099 \pm 0.014$ & $0.106 \pm 0.010$ & $\ldots$ & $\ldots$ & & $\ldots$ & $\ldots$ \\
\hline$f_{\nu} \ldots \ldots \ldots \ldots \ldots \ldots \ldots$ & & & & & $<0.10$ & $<0.04$ & . & & & & 0 \\
\hline$n_{s} \ldots \ldots \ldots \ldots \ldots$ & $0.96_{-0.04}^{+0.27}$ & $1.04_{-0.05}^{+0.06}$ & $1.02_{-0.07}^{+0.06}$ & $1.04_{-0.06}^{+0.05}$ & $1.02 \pm 0.05$ & $1.03 \pm 0.05$ & $0.99 \pm 0.06$ & $0.99 \pm 0.04$ & $0.97 \pm 0.03$ & $0.96 \pm 0.02$ & 0.96 \\
\hline$\tau$ & $<0.25$ & $<0.25$ & $0.16_{-013}^{+0.18}$ & $0.13_{-010}^{+0.13}$ & $\ldots$ & & $0.04^{+0.06}$ & $0.06 \pm 0.03$ & $0.14_{-006}^{+0.07}$ & $0.12_{-005}^{+0.06}$ & 0.12 \\
\hline$\Omega_{m} h \ldots \ldots \ldots \ldots \ldots$ & $\ldots$ & $0.19 \pm 0.02$ & & & $0.18 \pm 0.03$ & $0.19 \pm 0.02$ & $\ldots$ & & & & 0.185 \\
\hline 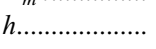 & $\ldots$ & $0.66_{-0.03}^{+0.09}$ & $0.55_{-0.09}^{+0.09}$ & $0.69_{-0.02}^{+0.02}$ & $0.67 \pm 0.05$ & $0.66 \pm 0.03$ & $0.71 \pm 0.13$ & $0.73 \pm 0.11$ & $0.73 \pm 0.05$ & $0.72 \pm 0.03$ & 0.72 \\
\hline
\end{tabular}

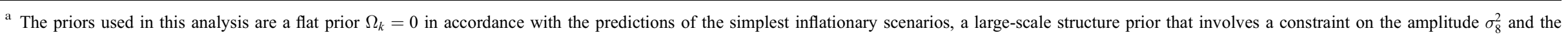
shape of the matter power spectrum, the HKP prior for $h$, and the SN Ia priors.

${ }^{\mathrm{b}}$ The priors used in this analysis are a flat prior $\Omega_{k}=0$ in accordance with the predictions of the simplest inflationary scenarios, the BBN prior for $\omega_{b}$, the HKP prior for $h$, and the SN Ia priors. 
A number of recent analyses combine information from a range of independent observational data sets (e.g., Efstathiou et al. 2002; Lewis \& Bridle 2002; Sievers et al. 2003; Wang et al. 2003; Spergel et al. 2003), enabling certain degeneracies of the individual data sets to be resolved. As the observational data become more precise and diverse, they form an increasingly tight network of parameter constraints. Constraints from a variety of astrophysical data, including CMB temperature (TT) and temperature-polarization (TE) angular power spectra, the Two-Degree Field Galaxy Redshift Survey (2dFGRS) power spectrum (Percival et al. 2001), Type Ia supernova (SN Ia) measurements of the angular diameterdistance relation (Garnavich et al. 1998; Riess et al. 2001), measurements of the Ly $\alpha$ forest power spectrum (Croft et al. 2002; Gnedin \& Hamilton 2002), Hubble Key Project (HKP) constraints on the Hubble parameter $h$ (Freedman et al. 2001), and big bang nucleosynthesis (BBN) constraints on the baryon fraction (Burles, Nollett, \& Turner 2001), are beginning to refine an observationally concordant cosmological model.

The results of recent CMB-only analyses and joint likelihood analyses are given in Table 1. The most current joint analysis to date suggests the observationally concordant cosmology (Spergel et al. 2003): $\Omega_{\kappa} \simeq 0, \Omega_{\Lambda} \simeq 0.7\left(\Omega_{m}=\right.$ $\left.\Omega_{b}+\Omega_{c} \simeq 0.3\right), \Omega_{b} h^{2} \simeq 0.0226, n_{s} \simeq 0.96, h \simeq 0.72$, and $\tau \simeq 0.12$ with $A_{t}$, and $\Omega_{\nu}$ taken to be zero. With more precise and diverse cosmological observations, the ability of this standard $\Lambda \mathrm{CDM}$ cosmology to describe the observational universe will be extended and tested for inconsistencies.

\subsection{Goodness of Fit of the Concordance Model to the CMB}

We perform a simple $\chi^{2}$ calculation (see Appendix A) to determine the goodness of fit of the concordance $\Lambda \mathrm{CDM}$ cosmology to the CMB, employing the band power temperature measurements of Table 2 and their associated window functions. We limit our analysis to $2<l<2000$ because secondary anisotropy contributions, such as the SunyaevZel'dovich effect (Sunyaev \& Zel'dovich 1970) and/or the signature of primordial voids (Griffiths, Kunz, \& Silk 2003), may dominate at $l>2000$. We bin the WMAP data into 70 bins, carefully chosen so as not to smooth out any genuine features in the data, following the method of Appendix B.

The model radiation angular power spectrum is calculated using CMBFAST (Seljak \& Zaldarriaga 1996). However, rather than adopting the CMBFAST COBE Differential Microwave Radiometer (DMR) normalization, we implement a numerical approximation to marginalization (see Appendix A) to find the optimal normalization of the theoretical model to the full observational data set. We also similarly treat the beam uncertainties of BOOMERANG98, MAXIMA1, and PyV and the calibration uncertainties associated with the observations as free parameters with Gaussian distributions (see eq. [A6]).

The minimized $\chi^{2}$ for the concordance model is 258 . In order to determine how good a fit this model is to the observational data, we need to know the number of degrees of freedom of the analysis. Although 274 degrees of freedom are provided by the number of observational data points (assuming that they are uncorrelated), these are reduced by the number of concordance parameters that are constrained using the CMB data alone. The flatness of the concordance model $\left(\Omega_{\kappa} \simeq 0\right.$ ), the tilt of the primordial power spectrum of scalar perturbations $\left(n_{s} \simeq 0.96\right)$, and the optical depth of reionization $(\tau \simeq 0.12)$ are extracted almost entirely from the CMB data. The remaining concordant parameters are strongly constrained by non-CMB observations. We therefore estimate that 3 degrees of freedom should be subtracted from the original 274.

Within our analysis we marginalize over a number of nuisance parameters. We fit for 22 individual calibration constants (all the CBI observations are assumed to shift together), three beam uncertainties (those of BOOMERANG98, MAXIMA1, and PyV), and an overall normalization. Thus, a further 26 degrees of freedom must be subtracted, leaving 245 degrees of freedom. The $\chi^{2}$ per degree of freedom is then $258 / 245=1.05$, indicating that the concordance cosmology provides a good fit to the CMB data alone.

Data correlations other than the correlated beam and calibration uncertainties of individual experiments, which we take to have no interexperiment dependence, are not considered in our analysis. Including such correlations would further reduce the number of degrees of freedom, increasing the $\chi^{2}$ per degree of freedom. However, our result is in agreement with joint likelihood analyses that find that the cosmological model that best fits the CMB data is a better fit at the 1 or $2 \sigma$ level than the fit to the concordance model (Wang et al. 2002).

We find the normalization of the concordance model to the full CMB data set to be $Q_{10}=18.56 \pm 0.04 \mu \mathrm{K}$, where $Q_{10}$ is defined through the relation (Lineweaver \& Barbosa 1998)

$$
10(10+1) C_{10}=\frac{24 \pi}{5} \frac{Q_{10}^{2}}{T_{\mathrm{CMB}}^{2}} .
$$

The normalized concordance model is plotted with the calibrated and beam-corrected observational data in Figure 1. It is

TABLE 2

The Current Compilation of CMB Observational Data from $l=2$ to 2000

\begin{tabular}{|c|c|c|c|c|c|c|c|}
\hline Experiment & References & $l_{\mathrm{eff}}$ & $l_{\min }$ & $l_{\max }$ & $\begin{array}{l}C_{l_{\mathrm{eff}}^{\mathrm{obs}}} \pm \sigma^{\mathrm{obs}} \\
\quad(\mu \mathrm{K})\end{array}$ & $\begin{array}{l}\sigma_{u}{ }^{\mathrm{a}} \\
(\%)\end{array}$ & $\begin{array}{l}\text { Publication Date } \\
\text { (yr) }\end{array}$ \\
\hline \multirow[t]{5}{*}{ ACBAR ........... } & 1 & 187.0 & 75.0 & 300.0 & $6767.0_{-1323.0}^{+1323.0}$ & 20.0 & 2002.9 \\
\hline & 1 & 389.0 & 307.0 & 459.0 & $2874.0_{-605.0}^{+605.0}$ & 20.0 & 2002.9 \\
\hline & 1 & 536.0 & 462.0 & 602.0 & $2716.0_{-498.0}^{+498.0}$ & 20.0 & 2002.9 \\
\hline & 1 & 678.0 & 615.0 & 744.0 & $2222.0_{-360.0}^{+360.0}$ & 20.0 & 2002.9 \\
\hline & 1 & 842.0 & 751.0 & 928.0 & $2300.0_{-355.0}^{+355.0}$ & 20.0 & 2002.9 \\
\hline
\end{tabular}

Notes.-Table 2 is published in its entirety in the electronic edition of the Astrophysical Journal. A portion is shown here for guidance regarding its form and content.

a The $1 \sigma$ calibration uncertainty in temperature, $\sigma_{u}$, is given as a percentage and allows the data points taken at the same time using the same instrument to shift upward or downward together. For observations that result in a single data point, $\sigma_{u}$ is not given since either it is not quoted in the literature or it has been treated by adding it in quadrature to the statistical error bars.

REFERENCES.-(1) Kuo et al. 2004. 


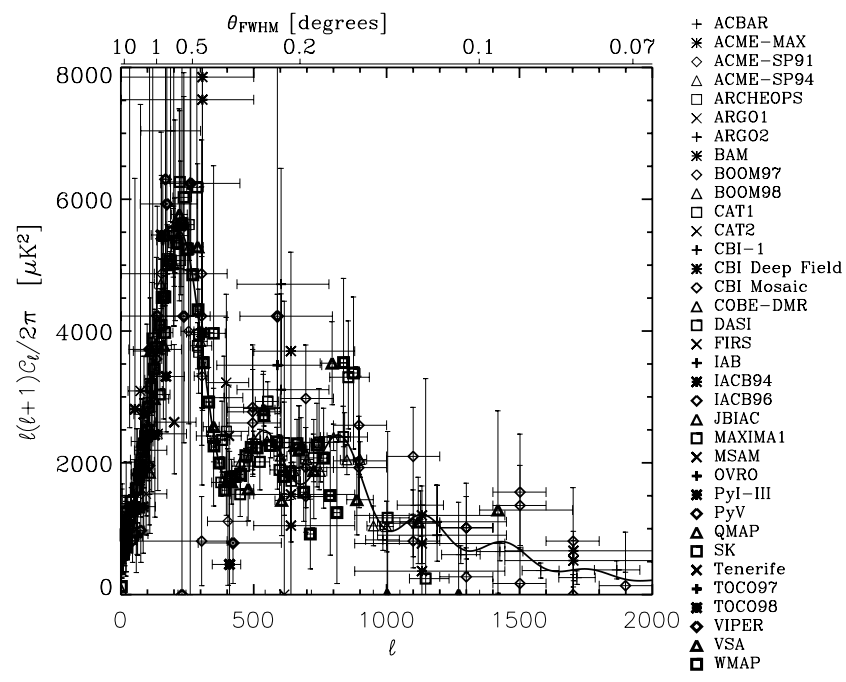

FIG. 1.-Concordance cosmology (Table 1) normalized to the full CMB data set, plotted with the recalibrated and (for BOOMERANG98, MAXIMA1, and PyV) beam-corrected $\mathrm{CMB}$ observational data (Table 2) that span the scales $2<l<2000$. [See the electronic edition of the Journal for a color version of this figure.]

difficult to distinguish the most important measurements because there are so many CMB data points on the plot and it is dominated by those with the largest error bars. Therefore, for clarity, we bin the data as described in Appendix B. The binned observations are plotted with the concordance cosmology in Figure 2.

\section{EXAMINING THE RESIDUALS}

Our analysis is based on the assumption that the combined cosmological observations used to determine the concordance model are giving us a more accurate estimate of cosmological parameters, and therefore of the true $C_{l}$ spectrum, than is given by the CMB data alone. Under this assumption, the residuals

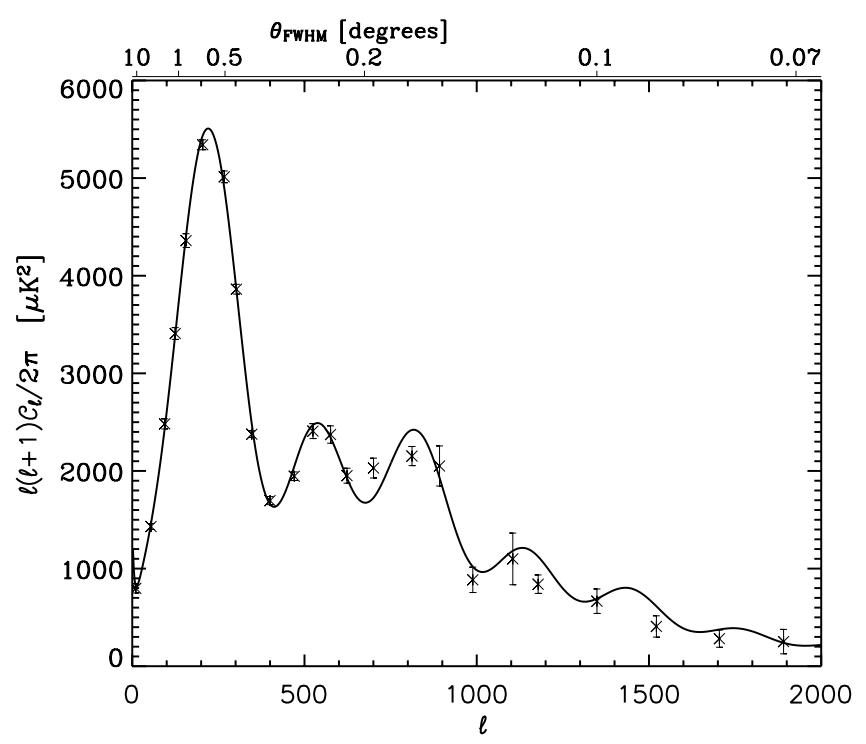

FIG. 2.-Concordance cosmology (Table 1) normalized to the full CMB data set, plotted with the binned observational data. The binning methodology is given in Appendix B. All statistical analyses detailed in this paper are performed on the raw, unbinned data. [See the electronic edition of the Journal for a color version of this figure.] of the individual observed CMB band powers and the concordance $\Lambda \mathrm{CDM}$ model become tools to identify a variety of systematic errors. To this end, we create residuals $R_{i}$ of the observed band power anisotropies $C_{l_{\mathrm{eff}}}^{\mathrm{obs}}(i) \pm \sigma^{\mathrm{obs}}(i)$ with respect to the concordant band powers $C_{l_{\mathrm{eff}}}^{\text {th }}(i)$ such that

$$
R_{i}=\frac{C_{l_{\mathrm{eff}}}^{\mathrm{obs}}(i)-C_{l_{\mathrm{eff}}}^{\mathrm{th}}(i)}{C_{l_{\mathrm{eff}}}^{\mathrm{th}}(i)} \pm \frac{\sigma^{\mathrm{obs}}(i)}{C_{l_{\mathrm{eff}}}^{\mathrm{th}}(i)} .
$$

Systematic errors are part of the CMB band power estimates at some level. We examine our data residuals as functions of the instrumental and observational details, listed in Table 3, that may be associated with systematic errors. Possible sources of systematic uncertainty are discussed in the following section. If the analysis determines that a linear trend can produce a significantly improved fit in comparison to that of a zero gradient line (zero line) through the data, it may be indicative of an unidentified systematic source of uncertainty. Similarly, any significant outliers may point to untreated systematics. Our results are summarized in Table 4.

The zero line through all the residual data gives a $\chi^{2}$ of 258. As previously discussed, the analysis that determines the goodness of fit of the concordance model to the CMB data has 245 degrees of freedom. A further 2 degrees of freedom must be subtracted for the line slope and intercept parameters that are varied in the line-fitting analysis, leaving 243 degrees of freedom. However, when the residuals are examined as a function of angular scale, the intercept of any line that fits the residual data will depend on the normalization of the concordance model. In this case, we therefore subtract only 1 further degree of freedom, giving 244 degrees of freedom.

The zero-line fit to the residual data has a $\chi^{2}$ per degree of freedom of 1.06 and a $76 \%$ probability of finding a line that better fits the data. In order to determine the significance of a better fit provided by a linear trend, an understanding of the statistical effects of introducing the two parameters to the line-fitting analysis is required. For a two-dimensional Gaussian distribution, the difference between the $\chi^{2}$ of the best-fit model and a model within the $68 \%$ confidence region of the best-fit model is less than 2.3 , and for a model that is within the $95 \%$ confidence region of the best-fit model, this difference is less than 6.17 (Press et al. 1992, p. 692). Our $68 \%$ and $95 \%$ contours in Figures $3-11$ are so defined. The further the horizontal concordance zero line is from the bestfitting slope, the stronger the indication of a possible systematic error.

\section{POSSIBLE SOURCES OF SYSTEMATIC UNCERTAINTY}

\subsection{Angular Scale-dependent Effects}

We examine scale-dependent uncertainties by plotting the residuals as a function of $l$ (Fig. 3). The shape of the window function is most critical when the curvature of the power spectrum is large (at the extrema of the acoustic oscillations). We therefore also explore the residuals as a function of the narrowness of the filter functions $\Delta l / l$.

The resolution of the instrument and the pointing uncertainty become increasingly important as fluctuations are measured at smaller angular scales. Small beams may be subject to unidentified smearing effects that may show up as a trend in the residual data with respect to $\theta_{\text {beam }} l_{\text {eff. }}$ Thus, we examine the residuals as a function of $\theta_{\text {beam }} l_{\text {eff }}$ (Fig. 10) and 
TABLE 3

Details of the CMB Observational Techniques

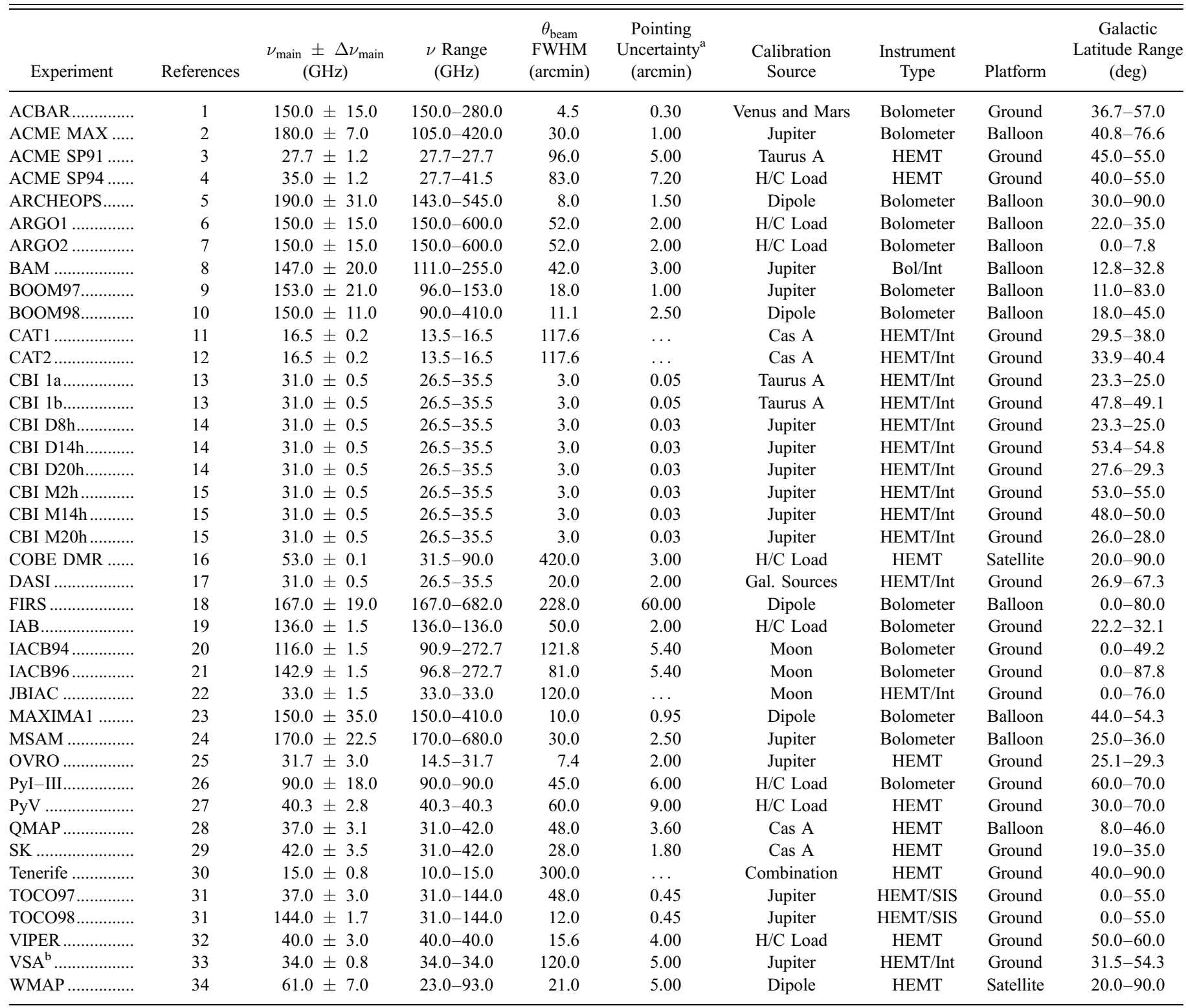

${ }^{a}$ Where pointing uncertainties are not given, they are not quoted in the literature.

b The VSA results quoted in the literature are the combined detections from a number of separate fields observed at various Galactic longitudes and latitudes. Information is not given in the literature to enable the contributions from the different fields to be separated. Therefore, the VSA Galactic longitude range is omitted. However, since the fields are not very dispersed in Galactic latitude, this range is listed.

References.-(1) Kuo et al. 2004; (2) Alsop et al. 1992; Lim et al. 1996; Tanaka et al. 1996; (3) Gunderson et al. 1995; (4) Benoît et al. 2003; (5) Ganga et al. 1997; Gunderson et al. 1995; (6) de Bernardis et al. 1993, 1994; (7) de Bernardis et al. 1993, 1994; Masi et al. 1995, 1996; (8) Tucker et al. 1997; (9) Mauskopf et al. 2000; Piacentini et al. 2002; (10) Crill et al. 2003; Netterfield et al. 2002; Ruhl et al. 2003; (11) Scott et al. 1996; (12) Baker et al. 1999; (13) Padin et al. 2001, 2002; (14) Mason et al. 2003; (15) Mason et al. 2003; Pearson et al. 2003; (16) Kogut et al. 1992, 1996; Tegmark \& Hamilton 1997; (17) Halverson et al. 2002; Leitch et al. 2002; (18) Page, Cheng, \& Meyer 1990; Meyer, Cheng, \& Page 1991; Ganga et al. 1994; (19) Piccirillo \& Calisse 1993; (20) Femenia et al. 1998; (21) Femenia et al. 1998; Romeo et al. 2001; (22) Dicker et al. 1999; Melhuish et al. 1999; (23) Lee et al. 2001; Hanany et al. 2000; (24) Fixsen et al. 1996; Wilson et al. 2000; (25) Leitch et al. 2000; (26) Dragovan et al. 1994; Ruhl et al. 1995; Platt et al. 1997; (27) Coble et al. 1999, 2003; (28) de Oliveira-Costa et al. 1998; Devlin et al. 1998; Herbig et al. 1998; Miller et al. 2002; (29) Netterfield et al. 1997; Miller et al. 2002; (30) Davies et al. 1996; Gutierrez et al. 2000; (31) Miller et al. 2002; (32) Peterson et al. 2000; (33) Grainge et al. 2003; Scott et al. 2003; Taylor et al. 2003; Watson et al. 2003; (34) Bennett et al. 2003 ; Hinshaw et al. 2003.

pointing uncertainty (Fig. 11) to look for hints of systematic errors associated with these factors.

\subsection{Foregrounds}

If foreground emission is present, it will raise the observed power. Galactic and extragalactic signals from synchrotron, bremsstrahlung, and dust emission have frequency dependencies that are different from that of the CMB (e.g., Tegmark
\& Efstathiou 1996). If such contamination is present in the data, it may be revealed by a frequency dependence of the residuals (Fig. 6).

Multiple frequency observations provide various frequency lever arms that allow individual groups to identify and correct for frequency-dependent contamination. Experiments with broad frequency coverage may be better able to remove this contamination than those with narrow frequency coverage. We 
TABLE 4

Results of the Residual Analyses

\begin{tabular}{|c|c|c|c|c|c|c|c|c|c|c|c|}
\hline Parameter & $l_{\mathrm{eff}}$ & $\begin{array}{c}|b| \\
\text { Range }^{\mathrm{a}}\end{array}$ & $\begin{array}{l}\text { Central } \\
\qquad|b|^{\mathrm{b}}\end{array}$ & $\nu_{\text {main }}$ & $\frac{\left(\nu_{\max }-\nu_{\min }\right)}{\nu_{\text {main }}}$ & $\begin{array}{c}\text { Calibration } \\
\text { Source }\end{array}$ & $\begin{array}{c}\text { Instrument } \\
\text { Type }\end{array}$ & $\theta_{\text {beam }} l_{\text {eff }}$ & $\begin{array}{c}\text { Pointing } \\
\text { Uncertainty }\end{array}$ & $\begin{array}{c}\text { Instrument } \\
\text { Platform }\end{array}$ & $\Delta l / l_{\mathrm{eff}}$ \\
\hline 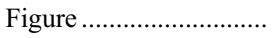 & 3 & 4 & 5 & 6 & 7 & 8 & 9 & 10 & 11 & $\ldots$ & $\ldots$ \\
\hline 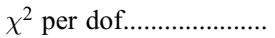 & 1.06 & 0.74 & 1.06 & 1.06 & 1.06 & 1.06 & 1.06 & 1.06 & 1.07 & 1.06 & 1.05 \\
\hline Significance $^{c}(\sigma) \ldots \ldots \ldots .$. & $\sim 0.5$ & $>3$ & $<0.5$ & $<0.5$ & $<0.5$ & $<1$ & $<0.5$ & $<0.5$ & $<0.5$ & $<0.5$ & $<2$ \\
\hline
\end{tabular}

a The $|b|$ range analysis involves a two-dimensional fit (see $\S 5$ ).

b The central $|b|$ analysis involves a one-dimensional fit (see $\S 5$ ).

c The significance of the deviation of the zero line from the best-fit linear model. As discussed in $\S 2.2$, correlations other than the correlated beam and calibration uncertainties of individual experiments are not considered in our analysis. Ignoring such correlations may result in the significance of the deviation being smaller than expected.

therefore examine the residuals as a function of the frequency lever arm $\left(\nu_{\max }-\nu_{\text {min }}\right) / \nu_{\text {main }}$ (Fig. 7).

Observations taken at lower absolute Galactic latitudes, $|b|$, will be more prone to Galactic contamination. We check for this effect by examining the residuals as a function of $|b|$ range (Fig. 4). In this case, we take $|b|$ to be the value central to the range observed and the $1 \sigma$ uncertainties in $|b|$ to extend to the extrema of this range (see Table 3 ). We also examine the residuals as a function of central $|b|$ neglecting the range in $|b|$ observed (Fig. 5). Using ranges in $|b|$ as statistical errors as in Figure 4 is problematic, but so too is treating the band powers as if they result from measurements at a precise value of $|b|$ (Fig. 5). The most plausible result is intermediate between these two cases.

\subsection{Calibration}

To analyze various experiments, knowledge of the calibration uncertainty of the measurements is necessary. Independent observations that calibrate off the same source will have calibration uncertainties that are correlated at some level and therefore a fraction of their freedom to shift upward

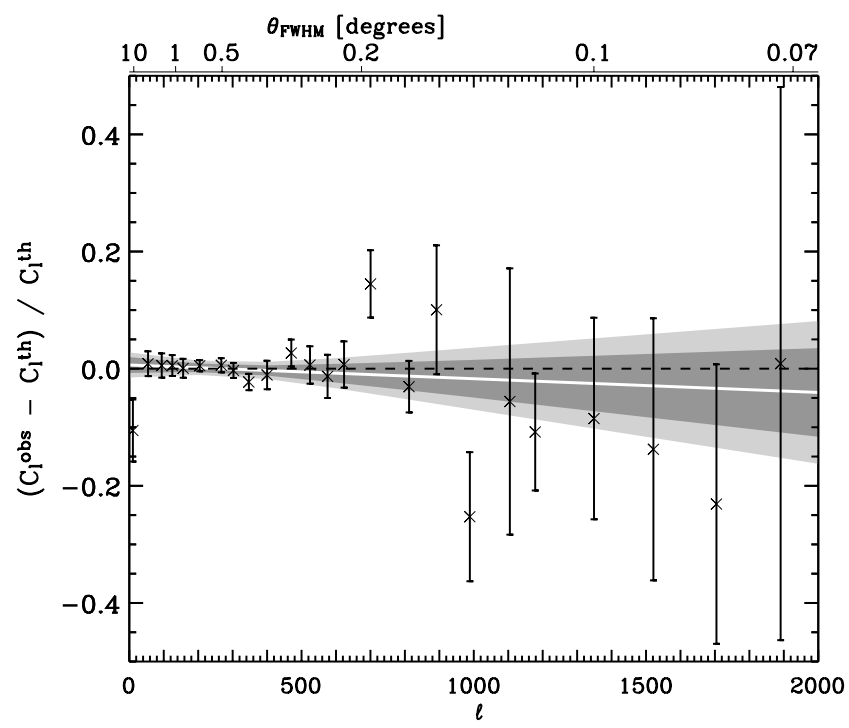

FIG. 3.-CMB data residuals plotted against $l$ (bottom $x$-axis) and angular scale (top $x$-axis). Although there is little evidence for a trend in this plot, the bins at $l>900$ are predominantly low, which may point to a marginal source of systematic error or a need to slightly dampen the small angular scale power in the concordance model. The $\chi^{2}$ per degree of freedom for the fit of the line to the data is 1.05 , so the best-fit line is a slightly better fit to the data than the zero line that has a $\chi^{2}$ per degree of freedom of 1.06. [See the electronic edition of the Journal for a color version of this figure.] or downward will be shared. For example, ACME MAX, BOOMERANG97, CBI, MSAM, OVRO, TOCO, and VSA all calibrate off Jupiter, so part of the quoted calibration uncertainties from these experiments will come from the brightness uncertainty of this source. Wang et al. (2002) perform a joint analysis of the CMB data making the approximation that the entire contribution to the calibration uncertainty from Jupiter's brightness uncertainty is shared by the experiments that use this calibration source. The true correlation will be lower since the independent experiments observed Jupiter at different frequencies.

Interexperiment correlations are not considered in our analysis, since we are unable to separate out the fraction of uncertainty that is shared by experiments. Instead, we test for any calibration-dependent systematics by examining the data residuals with respect to the calibration source (Fig. 8). We note that including correlations between data points would reduce the number of degrees of freedom of our $\chi^{2}$ analysis. The order of the calibration sources is arbitrary, so the fitting of a line serves only to verify that the line-fitting and confidence interval-determining codes are working as expected.

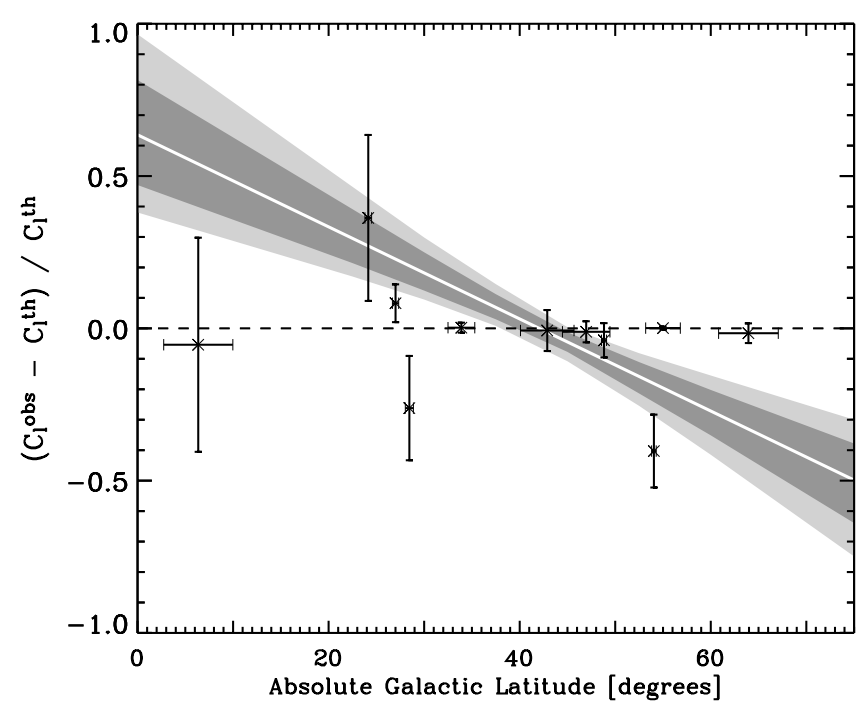

FIG. 4. - CMB data residuals plotted against absolute Galactic latitude $|b|$. The fitting routine uses uncertainties in both the $y$ - and $x$-directions and assumes that the uncertainty in $|b|$ extends to the limits of the $|b|$ range (Table 2). The $\chi^{2}$ per degree of freedom for the fit of the line to the data is $177 / 243=0.73$. There is a more than $3 \sigma$ trend in this regression plot that may be indicative of a systematic error associated with absolute Galactic latitude. [See the electronic edition of the Journal for a color version of this figure.] 


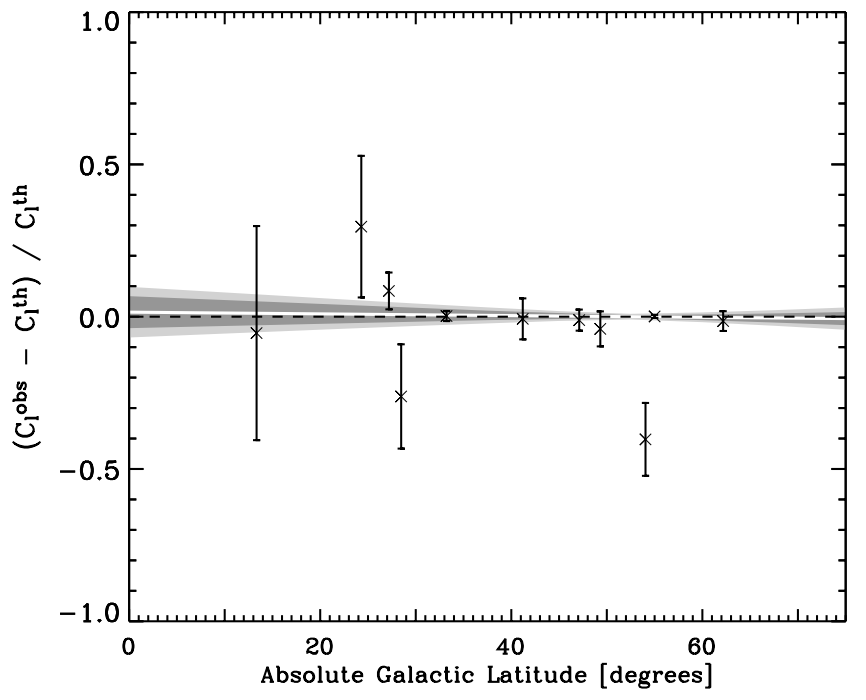

FIG. 5.-CMB data residuals plotted against the central absolute Galactic latitude $|b|$, neglecting the range in $|b|$ observed (i.e., the $x$-coordinate freedom has been removed from the fit of Fig. 4; see $\S 4.2$ ). The $\chi^{2}$ per degree of freedom for the fit of the line to the data is $258 / 243=1.06$. The greater than $3 \sigma$ trend of Fig. 4 is reduced to less than $0.5 \sigma$ here. The different methods used in these two plots are discussed in $\S 5$. [See the electronic edition of the Journal for a color version of this figure.]

However, any significant outliers may indicate unidentified calibration-dependent systematics.

\subsection{Instrument Type and Platform}

The experiments use combinations of three types of detector that operate over different frequency ranges. We classify the data with respect to their instrument type: HEMT interferometers (HEMT/Int), HEMT amplifier-based non-interferometricinstruments (HEMT), HEMT-based amplifier and SIS-based mixer combination instruments (HEMT/SIS), bolometric instruments, and bolometric interferometers (Bol/Int). We check for receiver-specific systematic effects by plotting the residuals as a function of instrument type (Fig. 10). Again

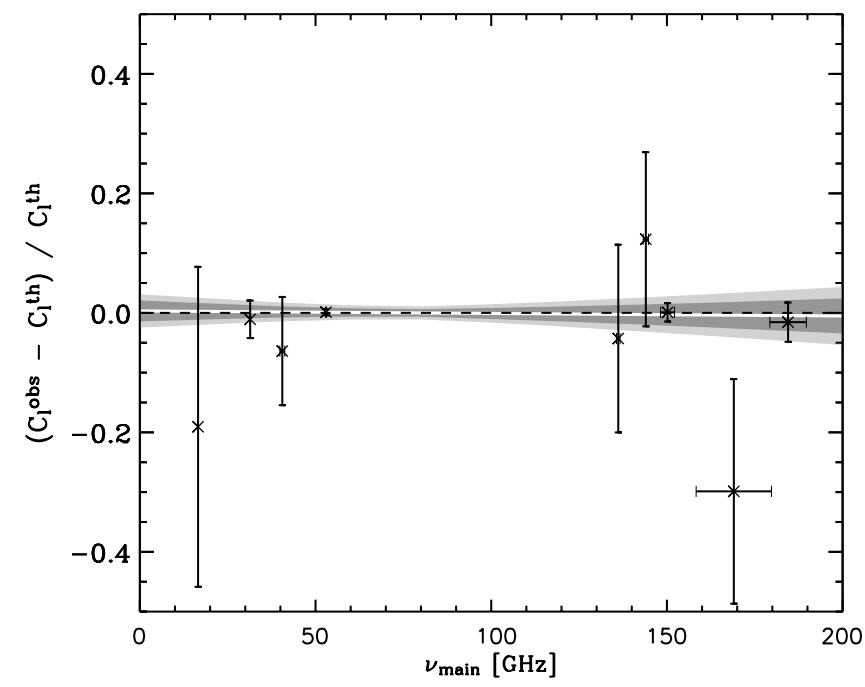

FIG. 6.-CMB data residuals plotted against the main frequency of individual instruments $\nu_{\text {main }}$. The fitting routine uses uncertainties in both the $y$ and $x$-directions. The $\chi^{2}$ per degree of freedom for the fit of the line to the data is $258 / 243=1.06$, so the best-fit line does not improve the fit beyond that of the zero line. Thus, there is no evidence for a trend in this regression plot. [See the electronic edition of the Journal for a color version of this figure.]

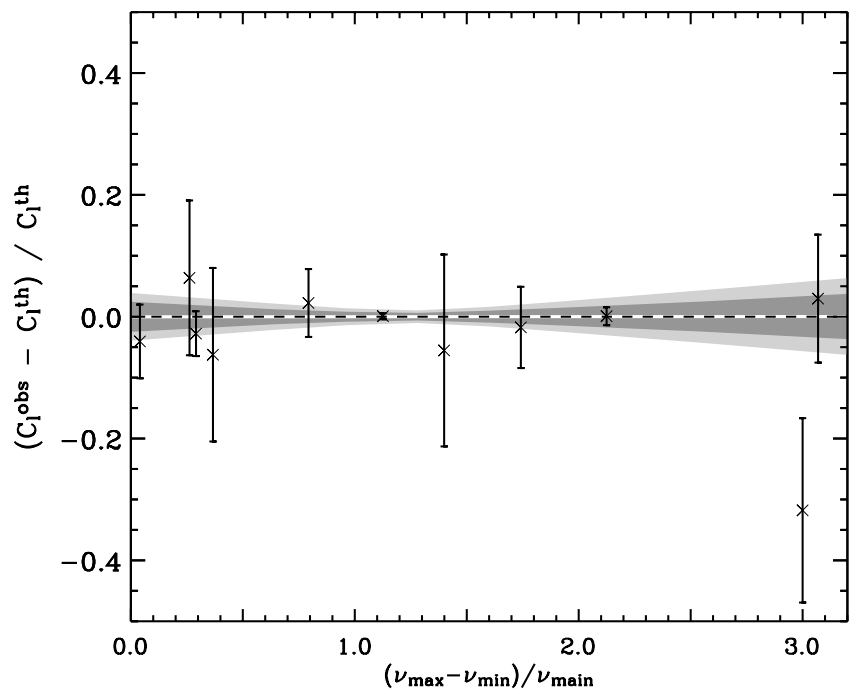

FIG. 7.- CMB data residuals plotted against the lever arm in frequency $\left(\nu_{\max }-\nu_{\min }\right) / \nu_{\operatorname{main}}$. The $\chi^{2}$ per degree of freedom for the fit of the line to the data is $258 / 243=1.06$, so the best-fit line does not improve the fit beyond that of the zero line. Thus, there is no evidence for a trend in this regression plot. [See the electronic edition of the Journal for a color version of this figure.]

the order we choose for instrument type is arbitrary, and it is significant outliers that we are interested in.

Water vapor in the atmosphere is a large source of contamination for ground-based instruments. There may also be systematic errors associated with the temperature and stability of the thermal environment. We therefore explore instrument platform dependencies of the data residuals. We choose to order the instrument platform according to altitude.

\subsection{Random Controls}

We use a number of control regressions to check that our analysis is working as expected. To this end, the residuals are

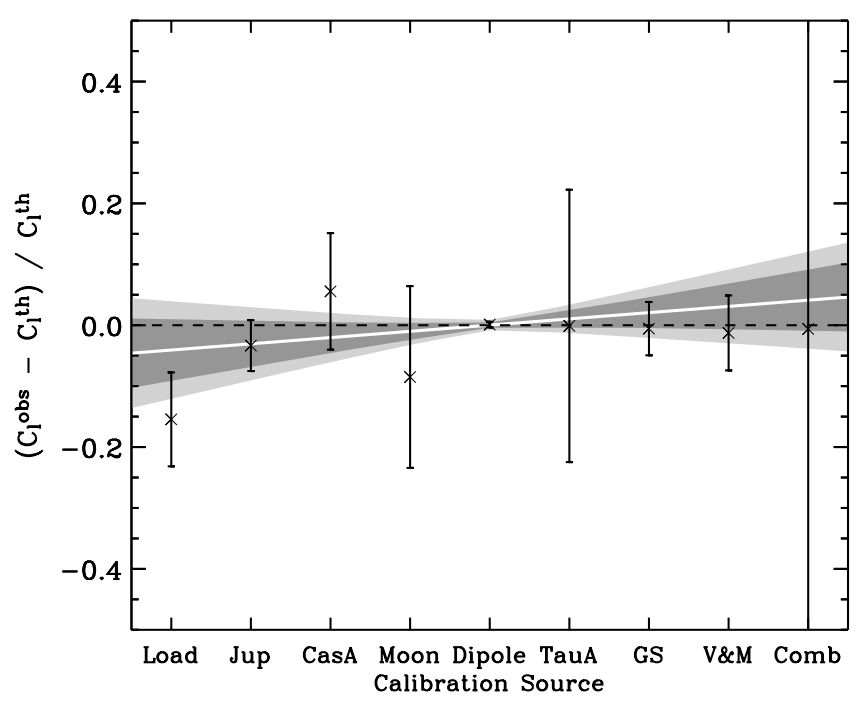

FIG. 8.- CMB data residuals plotted against calibration source. There are no large outliers. The order of the calibration sources is arbitrary, so the fitting of a line serves only to verify that the line-fitting and confidence intervaldetermining codes are working as expected. The zero line is just inside the border of the $68 \%$ confidence region for the best-fit line, confirming that we should not be suspicious of trends in our residuals that are revealed at less than the $1 \sigma$ level. [See the electronic edition of the Journal for a color version of this figure.] 


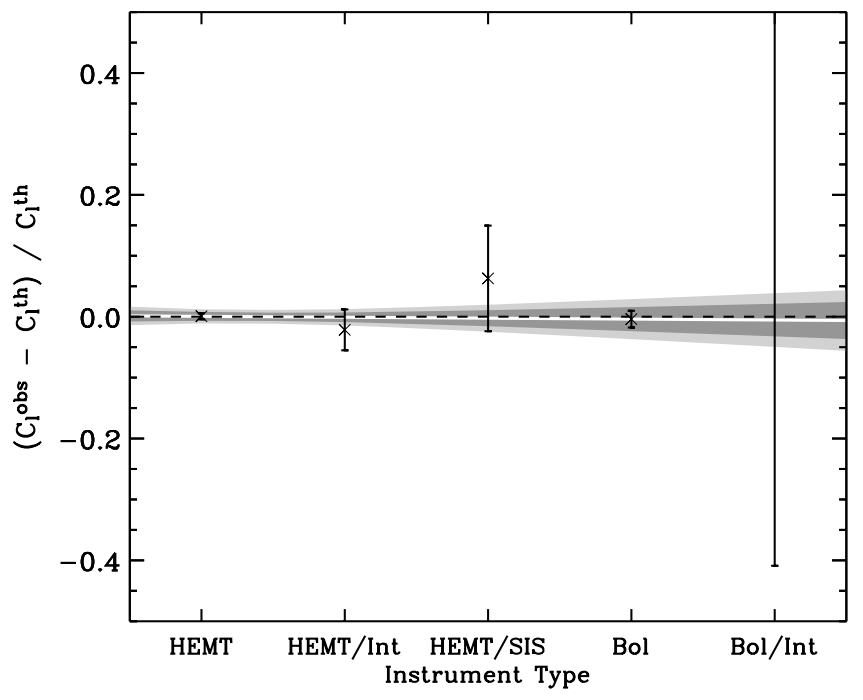

FIG. 9.-CMB data residuals plotted against instrument type. There are no large outliers. As in Fig. 8, the order of the instrument types is arbitrary, so the fitting of a line serves only to verify that the line-fitting and confidence interval-determining codes are working as expected. [See the electronic edition of the Journal for a color version of this figure.]

examined with respect to the publication date of the band power data, the number of letters in the first author's surname, and the affiliation of the last author. We expect the line fitted to these control regressions to be consistent with a zero line through the residual data. Any significant improvement provided by a linear fit to these residuals may be indicative of a problem in the software or methodology.

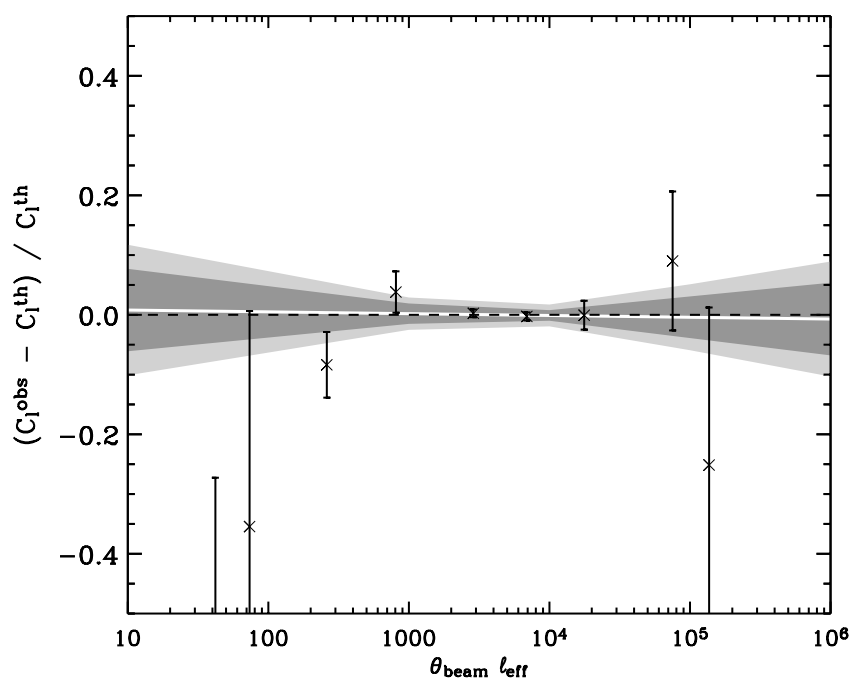

FIG. 10.-CMB data residuals plotted against $\theta_{\text {beam }} l_{\text {eff. The }} x$-axis is logarithmic so as to best display the data, and the residuals are examined for a linear trend with respect to this logarithmic axis. The $\chi^{2}$ per degree of freedom for the fit of the line to the data is $258 / 243=1.06$, so the best-fit line does not improve the fit beyond that of the zero line. The motivation for this plot is to see if there are any systematics associated with large beams sampling smallscale anisotropies (right side of plot) or with small beams sampling large-scale anisotropies (left side of plot). Small beams used to measure large angular scales may have stability problems analogous to the problems one runs into when trying to mosaic images together. Although no overall linear trend is observed, there is marginal evidence for power suppression at $\theta_{\text {beam }} l_{\text {eff }}<900$, suggesting that increased attention should be given to band power estimates in this regime. [See the electronic edition of the Journal for a color version of this figure.]

\section{RESULTS}

For the regressions plotted, the residual data are binned as described in Appendix B so that any trends can be more effectively visualized. Since the data-binning process may wash out any discrepancies between experiments, the linear fit analyses are performed on the unbinned data residuals. In Figures $3-11$, the line that best fits the data is plotted (solid white line) and the 68\% (dark grey region) and 95\% (light grey region) confidence regions of the best-fit line are shaded. For the plots for which it makes sense to test for a linear dependence, we report the $\chi^{2}$ per degree of freedom for the best-fit line and comment on the significance of the deviation of the zero line (black dashed line). For those plots for which the $x$-axis order is arbitrary, we comment on any significant outliers from the best-fit line.

Our results are summarized in Table 4. The lines fitted to our control regressions are consistent with a zero line through the residual data, suggesting that our line-fitting and confidence interval-determining codes are working as expected. We find a linear trend in the residuals with respect to the $|b|$ range of the observations (see Fig. 4). This trend is not eliminated by the removal of any one experiment and may be indicative of a source of Galactic emission that has not been appropriately treated.

In Figure 4 , the errors in both the $y$ - and $x$-directions are used in the fit. We have defined $|b|$ to be that of the center of the observations and the uncertainties to extend to the edges of the range. This allows the observations some freedom of the $x$ coordinate in the line-fitting analysis and weights heavily those detections that span small ranges in absolute Galactic latitude. It is therefore also interesting to examine the residuals with respect to the central $|b|$ to determine the significance of the trend with the $x$-coordinate freedom removed (see Fig. 5). The most plausible Galactic latitude regression will be somewhere between the regressions shown in these two plots.

Removing the $x$-coordinate freedom removes the significance of the trend. This result implies that experiments that

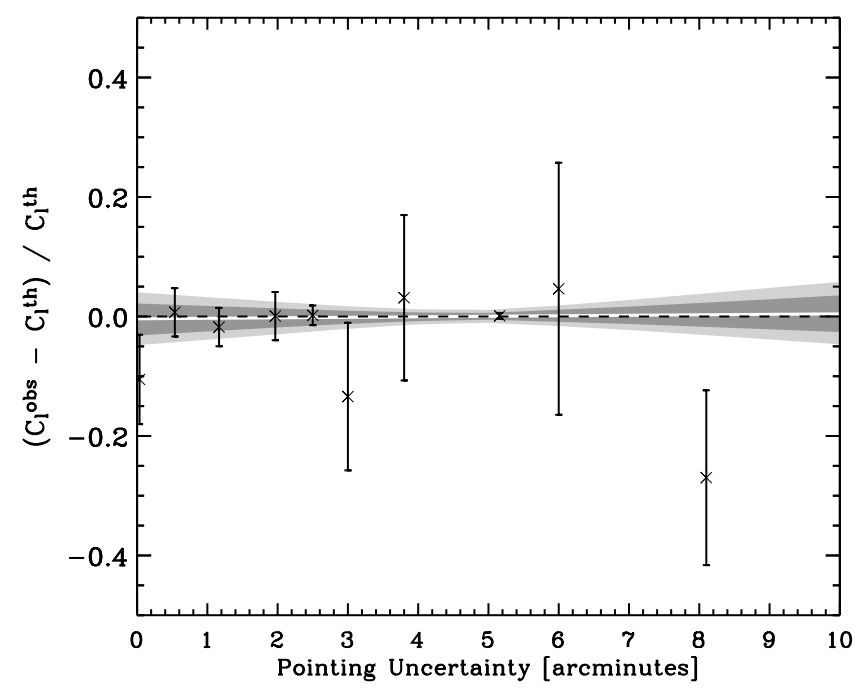

FIG. 11.-CMB data residuals plotted against pointing uncertainty. Six experiments do not quote pointing uncertainties and so are omitted from this analysis. With the six experiments omitted, there are 239 degrees of freedom. The $\chi^{2}$ per degree of freedom for both the zero line and the best-fitting line to the residual data is $256 / 239=1.07$, so the best-fit line does not improve the fit beyond that of the zero line. Thus, there is no evidence for a trend in this regression plot. [See the electronic edition of the Journal for a color version of this figure.] 
observe over small ranges in Galactic latitude are dominating the trend, and we therefore cannot simply correct for the systematic that is implied in Figure 4. The comparison of rms levels in Galactic dust (Finkbeiner, Davis, \& Schlegel 1999) and synchrotron ${ }^{1}$ maps over the areas of CMB observations may help to clarify the interpretation of the trend. Such a technique has recently been applied to the MAXIMA1 data (Jaffe et al. 2003) but has yet to be performed on the full CMB data set.

Other plots also show some evidence for systematic errors. Figures 2 and 3 indicate that 6 bins at $l>900$ prefer a lower normalization. This may be due to a systematic calibration error for some of the experiments in this $l$ range, underestimates of beam sizes or pointing uncertainties, or unidentified beam smearing effects at high $l$ for small beams. Although Figures 8 and 11 show little evidence for any trends, Figure 10 shows marginal evidence for power suppression at low $\theta_{\text {beam }} l_{\text {eff. }}$ The motivation for this plot is to see if there are any systematics associated with large beams sampling smallscale anisotropies (right side of plot) or with small beams sampling large-scale anisotropies (left side of plot). Small beams used to measure large angular scales may have stability problems analogous to the problems one runs into when trying to mosaic images together. Although no overall linear trend is observed, the trend indicated for $\theta_{\text {beam }} l_{\text {eff }}<900$ suggests that increased attention should be given to band power estimates in this regime.

\footnotetext{
${ }^{1}$ See http://astro.berkeley.edu/dust.
}

\section{SUMMARY}

Over the past 10 years, successive independent and semiindependent data sets have extended the angular scale, calibration precision, and freedom from Galactic contamination of the CMB power spectrum. Each CMB measurement contains useful cosmological information, and no data set is immune to contamination. It is therefore important to compare data sets and check for systematics. We have collectively tested the full CMB data set for inconsistencies with the concordance model, and our results indicate that the model is consistent with the data, although a need to slightly dampen power in the model at high $l$ is indicated (Fig. 3).

We have explored residuals of the observational data with respect to the concordance model to see if any patterns emerge that may indicate a source of systematic error. We have found little significant evidence for interexperiment inconsistencies other than a trend associated with Galactic latitude that may be an indication of low-level Galactic contamination of CMB observations made closer to the Galactic plane (Fig. 4). A more detailed comparison of CMB fields of observations with Galactic dust and synchrotron maps will be necessary to clarify the source of this trend.

L. M. G. thanks Martin Kunz for useful discussions and is grateful to the University of Sussex, where part of the work was carried out. L. M. G. acknowledges support from the Royal Society and PPARC. C. H. L. acknowledges a research fellowship from the Australian Research Council.

\section{APPENDIX A}

\section{$\chi^{2}$ MINIMIZATION METHODOLOGY}

For the observational power spectrum data quoted in the literature, individual $C_{l}$ values are not estimated; rather, band powers $C_{l_{\text {eff }}}$ are given that average the power spectrum through a filter, or window function. Each theoretical model must therefore be reexpressed in the same form before a statistical comparison can be made. This can be done using the method of Lineweaver et al. (1997).

Boltzmann codes such as CMBFAST (Seljak \& Zaldarriaga 1996) output theoretical power spectra in the form

$$
d_{1}(l)=\frac{l(l+1)}{2 \pi} C_{l}^{\text {theory }} \times \text { normalization. }
$$

Since the $C_{l}$ values are adimensional, they are multiplied by $T_{\mathrm{CMB}}^{2} \simeq(2.725 \mathrm{~K})^{2}$ (Mather et al. 1999) to express them in kelvin,

$$
d_{2}(l)=T_{\mathrm{CMB}}^{2} d_{1}(l) .
$$

The sensitivity of each observation (denoted $i$ ) to a particular $l$ is incorporated using the observational window function $W_{l}$,

$$
d_{3}(i, l)=d_{2}(l) \frac{(2 l+1) W_{l}^{i}}{2 l(l+1)} .
$$

The contribution from the model to the $i$ th observational band power is determined and the influence of the window function removed,

$$
C_{l_{\text {eff }}}^{\text {th }}(i)=\frac{\sum_{l=2}^{l_{\max }} d_{3}(i, l)}{I(i)}
$$

where $I(i)$ is the logarithmic integral of the window function,

$$
I(i)=\sum_{l=2}^{l_{\max }} \frac{(2 l+1) W_{l}^{i}}{2 l(l+1)} .
$$

Then $C_{l_{\mathrm{eff}}}^{\mathrm{th}}(i)$ can be statistically compared with the $i$ th band power measurement $C_{l_{\mathrm{eff}}}^{\mathrm{obs}}(i)$ given in Table 2 . 
The assumption that the CMB signal is a Gaussian random variable enables analysis via a likelihood procedure. As a result of the non-Gaussian distribution of the uncertainty in the band power measurements, an accurate calculation of the likelihood function $L$ is nontrivial. However, approximations to the true likelihood have been derived (Bond, Jaffe, \& Knox 2000; Bartlett et al. 2000). For example, the Bond et al. (2000) offset lognormal formalism is implemented in the publicly available RADPACK package. Unfortunately, the information necessary to implement this formalism has not yet been published by all observational groups. Therefore, in order to statistically analyze the complete CMB observational data set, we make the assumption that $L$ is Gaussian in $C_{l_{\mathrm{eff}}}$. Then,

$$
\chi^{2} \equiv-2 \ln L=\sum_{i}\left[\frac{C_{l_{\mathrm{eff}}}^{\mathrm{th}}(i)-C_{l_{\mathrm{eff}}}^{\mathrm{obs}}(i)}{\sigma^{\mathrm{obs}}(i)}\right]^{2} .
$$

The normalization of the primordial power spectrum is not predicted by inflationary scenarios and therefore the normalization of the concordance model to the full CMB observational data set is a free parameter. Unless we are particularly interested in the amplitude of primordial fluctuations, we can treat the model normalization $A$ as a nuisance parameter. Assuming a Gaussian likelihood, marginalization can be approximated numerically for the power spectrum normalization by computing the $\chi^{2}$ statistic of the concordance model for a number of discrete steps over the normalization range. The normalization that minimizes the $\chi^{2}$ can thereby be determined for a particular theoretical model.

The CMB measurements have associated calibration uncertainties (see Table 2) that allow data from the same instrument that is calibrated using the same source to shift collectively upward or downward. The observational band powers are multiplied by a calibration factor $U$ that can be treated as a nuisance parameter with a Gaussian distribution about 1 . This introduces an additional $\chi^{2}$ term to equation (A6) for each experiment that has an associated calibration uncertainty (see eq. [A7]).

Additionally, the BOOMERANG98, MAXIMA1, and PyV data sets have quantified beam and/or pointing uncertainties. The combined beam plus pointing uncertainty for each experiment introduces an additional term to equation (A6) that is a function of $B$. Here $B$ can be treated as a nuisance parameter, with a Gaussian distribution in $B \sigma_{b}(i)$ about 0 (see eq. [A7]). Lesgourgues \& Liddle (2001) give fitting functions for the combined beam plus pointing uncertainty in $D_{i}^{\text {obs }}$ for the BOOMERANG98 and MAXIMA1 experiments: $\sigma_{b, l}=0.43 \times 10^{-6} l^{2}$ for BOOMERANG98 and $\sigma_{b, l}=10^{-6} l^{1.7}$ for MAXIMA1. The $1 \sigma$ beam uncertainty for PyV is $\sigma_{b, l}=\exp ( \pm l(0.425)(0.015)(\pi / 180))-1$ (Coble et al. 2003).

The nuisance parameters are incorporated into equation (A6) to give

$$
\chi^{2}=\sum_{k}\left(\sum_{i=1}^{i_{\max }(k)}\left\{\frac{A C_{l_{\mathrm{eff}}}^{\mathrm{th}}(i)-\left[U(k)+B(k) \sigma_{b}(i)\right] C_{l_{\mathrm{eff}}}^{\mathrm{obs}}(i)}{\sigma(i)}\right\}^{2}+\left[\frac{U(k)-1}{\sigma_{u}(k)}\right]^{2}+[B(k)]^{2}\right),
$$

where the sum on $k$ is over the number of independent observational data sets.

\section{APPENDIX B}

\section{OBSERVATIONAL DATA BINNING}

The ever-increasing number of CMB anisotropies has made data plots such as Figure 1 difficult to interpret. The solution is to compress the data in some way. Many of the more recent analyses have chosen to concentrate on the data from just one or two experiments, often the most recently released. However, this not only neglects potentially useful information but can also unwittingly give more weight to particular observations that may suffer from systematic effects. We therefore choose to analyze all the available data.

One way to compress the data is to average them together into single band power bins in $l$-space. Such an approach has been taken by a number of authors (e.g., Knox \& Page 2000; Jaffe et al. 2001; Wang et al. 2002). Providing that the uncertainty in the data is Gaussian and correlations between detections are treated appropriately, narrow band power bins can be chosen that will retain all cosmological information.

Band power measurements from independent observations that overlap in the sky will be correlated to some extent. Such correlations can only be treated by jointly analyzing the combined overlapping maps to extract band power estimates that are uncorrelated or have explicitly defined correlation matrices. This process of data compression will wash out any systematics associated with a particular data set, so data consistency checks are vital before this stage. If the map data are unavailable, the crude assumption that independent observations are uncorrelated in space must be made. This assumption is made in likelihood analyses performed on the full power spectrum data set, and, since inclusion of these correlations would reduce the degrees of freedom of an analysis, the goodness of fit of a particular model to the data is better than it should be.

Some observational groups publish matrices encoding the correlations of their individual band power measurements. To some extent, the calibration uncertainties of experiments that calibrate using the same source are also correlated. Bond et al. (2000) describe a data-binning technique that takes a lognormal noise distribution that is approximately Gaussian and incorporates the correlation weight matrices of individual experiments. Wang et al. (2002) detail a method to treat partial correlations of calibration uncertainties. Both are useful to produce statistically meaningful data bins.

Data binning averages out any evidence for discrepancies between independent observations, and, in practice, data uncertainties are rarely Gaussian and the information required to treat correlated data is not always available. So although data binning is useful for visualization purposes, statistical analyses of the binned observations will generally give different results from those performed on the raw data. 
The statistical analyses detailed in this paper are performed on the published CMB band power measurements (Table 2). Binned data plots are presented purely to aid the interpretation of results. Therefore, each calibrated and (for BOOMERANG98, MAXIMA1, and PyV) beam-corrected observational data point is binned assuming it to be entirely uncorrelated. Bin widths must be carefully chosen so that important features of the data are not smoothed out, especially in regions of large curvature. For example, in the case of the power spectrum, an unwisely chosen bin that spans an acoustic maximum will average out the power in the bin to produce a binned data point that misleadingly assigns less power to the peak.

The contribution from the $i$ th observational measurement $\left(x_{i} \pm \sigma_{x, i}, y_{i} \pm \sigma_{y, i}\right)$ to a binned point $\left(x_{b} \pm \sigma_{x, b}, y_{b} \pm \sigma_{y, b}\right)$ is inverse variance weighted,

$$
\begin{aligned}
& x_{b} \pm \sigma_{x, b}=\frac{\sum_{i} x_{i} \sigma_{x, i}^{-2}}{\sum_{i} \sigma_{x, i}^{-2}} \pm \sqrt{\frac{1}{\sum_{i} \sigma_{x, i}^{-2}}}, \\
& y_{b} \pm \sigma_{y, b}=\frac{\sum_{i} y_{i} \sigma_{y, i}^{-2}}{\sum_{i} \sigma_{y, i}^{-2}} \pm \sqrt{\frac{1}{\sum_{i} \sigma_{y, i}^{-2}}} .
\end{aligned}
$$

If quoted error bars are asymmetric, a first guess for the binned data point is obtained by averaging the uncertainties. A more accurate estimate can then be converged upon by iterating over the binning routine, inserting the positive variance for measurements that are below the bin-averaged point and negative variance for those that are above.

All the data from a particular experiment will be measured using the same instrument and therefore can be binned together for the purpose of visualizing any trends in the data residuals with respect to the instrument design. When data from the same experiment are placed in the same bin, the variance of the resultant binned data point can be easily adjusted to account for any correlated calibration uncertainty associated with the observational data. A degree of freedom is reduced for each independent calibration uncertainty. This effectively tightens the constraints on the binned data.

For example, if $n$ calibrated data points in a bin have equal variance $\sigma_{y}$ and an entirely correlated calibration uncertainty, they share $n-1$ degrees of freedom and their contribution to the variance of the binned data point is then $\sigma_{y} /(n-1)^{1 / 2}$. When each of the $n$ data points has a different variance, their contribution to the uncertainty in the binned data point is given by

$$
\sigma_{y, b}=\frac{1}{\sqrt{\sum_{j} \sigma_{y, j}^{-2}-\left(n / \sum_{j} \sigma_{y, j}\right)^{2}}} .
$$

This method of binning is employed when appropriate to produce the plotted residual data bins.

Alsop, D. C., et al. 1992, ApJ, 395, 317

Baker, J. C., et al. 1999, MNRAS, 308, 1173

Bartlett, J. G., Douspis, M., Blanchard, A., \& Le Dour, M. 2000, A\&AS, 146,507

Bennett, C. L., et al. 2003, ApJ, 583, 1

Benoît, A., et al. 2003, A\&A, 399, L19

Bond, J. R., Jaffe, A. H., \& Knox, L. 2000, ApJ, 533, 19

Burles, S., Nollett, K. M., \& Turner, M. S., 2001, ApJ, 552, L1

Coble, K., et al. 1999, ApJ, 519, L5 2003, ApJ, 584, 585

Crill, B. P., et al. 2003, ApJS, 148, 527

Croft, R. A. C., Weinberg, D. H., Katz, N., \& Hernquist, L. 2002, ApJ, 581, 20

Davies, R. D., et al. 1996, MNRAS, 278, 883

de Bernardis, P., et al. 1993, A\&A, 271, 683

1994, ApJ, 422, L33

de Oliveira-Costa, A., Devlin, M. J., Herbig, T., Miller, A. D., Netterfield, C. B., Page, L. A., \& Tegmark, M. 1998, ApJ, 509, L77

Devlin, M. J., de Oliveira-Costa, A., Herbig, T., Miller, A. D., Netterfield,

C. B., Page, L. A., \& Tegmark, M. 1998, ApJ, 509, L69

Dicker, S. R., et al. 1999, MNRAS, 309, 750

Dragovan, M., Ruhl, J. E., Novak, G., Platt, S. R., Pernic, R., \& Peterson, J. R. 1994, ApJ, 427, L67

Efstathiou, G., \& Bond, J. R. 1999, MNRAS, 304, 75

Efstathiou, G., et al. 2002, MNRAS, 330, L29

Femenia, B., Rebolo, R., Gutierrez, C. M., Limon, M., \& Piccirillo, L. 1998, ApJ, 498, 117

Finkbeiner, D. P., Davis, M., \& Schlegel, D. J. 1999, ApJ, 524, 867

Fixsen, D. J., et al. 1996, ApJ, 470, 63

Freedman, W. L., et al. 2001, ApJ, 553, 47

Ganga, K., Page, L., Cheng, E., \& Meyer, S. 1994, ApJ, 432, L15

Ganga, K., Ratra, B., Gundersen, J. O., \& Sugiyama, N. 1997, ApJ, 484, 7

Garnavich, P. M., et al. 1998, ApJ, 509, 74

Gnedin, N. Y., \& Hamilton, J. J. S. 2002, MNRAS, 334, 107

Grainge, K., et al. 2003, MNRAS, 341, L23

\section{REFERENCES}

Griffiths, L. M., Kunz, M., \& Silk, J. 2003, MNRAS, 339, 680

Gunderson, J. O., et al. 1995, ApJ, 443, L57

Gutierrez, C. M., Rebolo, R., Watson, R. A., Davies, R. D., Jones, A. W., \& Lasenby, A. N. 2000, ApJ, 529, 47

Halverson, N. W., et al. 2002, ApJ, 568, 38

Hanany, S., et al. 2000, ApJ, 545, L5

Herbig, T., de Oliveira-Costa, A., Devlin, M. J., Miller, A. D., Page, L. A., \& Tegmark, M. 1998, ApJ, 509, L73

Hinshaw, G., et al. 2003, ApJS, 148, 135

Jaffe, A., et al. 2001, Phys. Rev. Lett., 86, 3475 2003, ApJ, submitted (astro-ph/0301077)

Knox, L., \& Page, L. 2000, Phys. Rev. Lett., 85, 1366

Kogut, A., et al. 1992, ApJ, 401, 1 1996, ApJ, 470, 653

Kuo, C. L., et al. 2004, ApJ, 600, 32

Lee, A. T., et al. 2001, ApJ, 561, L1

Leitch, E. M., et al. 2000, ApJ, 532, 37 2002, ApJ, 568, 28

Lesgourgues, J., \& Liddle, A. R. 2001, MNRAS, 327, 1307

Lewis, A., \& Bridle, S. 2002, Phys. Rev. D, 66, 103511

Lim, M. A., et al. 1996, ApJ, 469, L69

Lineweaver, C. H. 1997, in Microwave Background Anisotropies, ed. F. R. Bouchet, R. Gispert, B. Guiderdoni, \& J. Tran Thanh Van (Gif-sur-Yvette: Editions Frontieres), 69 1998, ApJ, 505, L69

Lineweaver, C. H., \& Barbosa, D. 1998, ApJ, 496, 624

Lineweaver, C. H., Barbosa, D., Blanchard, A., \& Bartlett, J. G. 1997, A\&A, 322,365

Lineweaver, C. H., et al. 1995, ApJ, 448, 482

Masi, S., et al. 1995, ApJ, 452, 253 1996, ApJ, 463, L47

Mason, B. S., et al. 2003, ApJ, 591, 540

Mather, J. C., Fixsen, D. J., Shafer, R. A., Mosier, C., \& Wilkinson, D. T. 1999, ApJ, 512, 511 
Mauskopf, P. D., et al. 2000, ApJ, 536, L59

Melhuish, S. J., et al. 1999, MNRAS, 305, 399

Meyer, S. S., Cheng, E., \& Page, L. A. 1991, ApJ, 371, L7

Miller, A., et al. 2002, ApJS, 140, 115

Netterfield, C. B., Devlin, M. J., Jarosik, N., Page, L., \& Wollack, E. J. 1997, ApJ, 474, 47

Netterfield, C. B., et al. 2002, ApJ, 571, 604

Padin, S., et al. 2001, ApJ, 549, L1 2002, PASP, 114, 83

Page, L. A., Cheng, E., \& Meyer, S. S. 1990, ApJ, 355, L1

Pearson, T. J., et al. 2003, ApJ, 591, 556

Percival, W. J., et al. 2001, MNRAS, 327, 1297

Peterson, J. B., et al. 2000, ApJ, 532, L83

Piacentini, F., et al. 2002, ApJS, 138, 315

Piccirillo, L., \& Calisse, P. 1993, ApJ, 411, 529

Platt, S. R., Kovac, J., Dragovan, M., Peterson, J. B., \& Ruhl, J. E. 1997, ApJ, 475, L1

Press, W. H., Teukolsky, S. A., Vetterling, W. T., \& Flannery, B. P. 1992, Numerical Recipes in Fortran 77: The Art of Scientific Computing (2d ed; Cambridge: Cambridge Univ. Press)

Riess, A. G., et al. 2001, ApJ, 560, 49

Romeo, G., Ali, S., Femenia, B., Limon, M., Piccirillo, L., Rebelo, R., \& Schaefer, R. 2001, ApJ, 548, L1
Ruhl, J. E., Dragovan, M., Platt, S. R., Kovac, J., \& Novak, G. 1995, ApJ, 453, L1

Ruhl, J. E., et al. 2003, ApJ, 599, 786

Scott, P. F., et al. 1996, ApJ, 461, L1 2003, MNRAS, 341, 1076

Seljak, U., \& Zaldarriaga, M. 1996, ApJ, 469, 437

Sievers, J. L., et al. 2003, ApJ, 591, 599

Spergel, D. N., et al. 2003, ApJS, 148, 175

Sunyaev, R. A., \& Zel'dovich, Ya. B. 1970, Ap\&SS, 7, 3

Tanaka, S. T., et al. 1996, ApJ, 468, L81

Taylor, A. C., et al. 2003, MNRAS, 341, 1066

Tegmark, M., \& Efstathiou, G. 1996, MNRAS, 281, 1297

Tegmark, M., \& Hamilton, A. J. S. 1997, preprint (astro-ph/9702019)

Tucker, G. S., Gush, H. P., Halpern, M., Shinkoda, I., \& Towlson, W. 1997, ApJ, 475, L73

Wang, X., Tegmark, M., Jain, B., \& Zaldarriaga, M. 2003, Phys. Rev. D, 68,123001

Wang, X., Tegmark, M., \& Zaldarriaga, M. 2002, Phys. Rev. D, 65, 123001

Watson, R. A., et al. 2003, MNRAS, 341, 1057

Wilson, G. W., et al. 2000, ApJ, 532, 57

Xu, Y., Tegmark, M., de Oliveira-Costa, A., Devlin, M. J., Herbig, T., Miller, A. D., Netterfield, C. B., \& Page, L. 2001, Phys. Rev. D, 63, 103002 